\title{
The Advantages of Partial Dentures Made of Cast Framework Cr-Co toward those Made of Resin Based Dentures
}

\author{
Gjergj BOÇARI $^{1, *}$, Neada HYSENAJ ${ }^{1}$, Alesio BOÇARI ${ }^{2}$ \\ ${ }^{1}$ Univeristy Dental Clinic, Tirana, Albania \\ ${ }^{2}$ Private Dental Clinic “Nobel”, Tirana, Albania \\ *Corresponding author: gjbocari@yahoo.com
}

Received March 26, 2014; Revised April 12, 2014; Accepted April 13, 2014

\begin{abstract}
Many investigations have shown that the cooperation of the denture design between the dental clinic and laboratory is often inadequate, with many dentists leaving it to the technician to decide the denture design. The aim of this study was to compare the stability of the removable partial dentures, made of cast framework base of CrCo toward those from acrylic resin-base. Through this study, we show the importance of a good planning and design of RDP, the evaluation of direct retentions, especially those of clasps and precise attachments for their impact on the longevity of the supporting teeth and tissues in different clinical cases, classified by Kennedy. The removable partial dentures evaluated in the study are either with or without a cast framework. In this study, are taken into observation 65 prostheses from 53 patients (28 maxillary and 37 mandibular) aged 38-70 years old. We have analyzed the type of clasps used and evaluated mobility scale and periodontal and clinical conditions of the abutment teeth in each case. After clinical examination we concluded that the abutment teeth where correct choice of clasp types were applied, had a mobility scale within physiological parameters and overall better clinical conditions compared to the abutment teeth were different type of clasps were used.
\end{abstract}

Keywords: direct retainers, denture design, Kennedy classification

Cite This Article: Gjergj BOÇARI, Neada HYSENAJ, and Alesio BOÇARI, "The Advantages of Partial Dentures Made of Cast Framework Cr-Co toward those Made of Resin Based Dentures." International Journal of Dental Sciences and Research, vol. 2, no. 2 (2014): 32-35. doi: 10.12691/ijdsr-2-2-2.

\section{Introduction}

T Nowadays, the scientific research is more focused on the fixed prosthetics and mainly on dental implants. Implant supported dentures are becoming popular for prosthetic restorations in partially edentulous patients. But however, removable partial denture (RPD) is still the primary treatment option from the patients' view because an implant-supported dentures are relatively expensive from a financial point of view and require a surgical procedure that not in every case can be done, and a longer treatment time compared to treatment with removable partial denture. As we know, a partial denture is supported by the abutment teeth and residual ridges which have different support characteristics while being under pressure. When a force is applied at the base of the saddle it generates rotational movements of the RPD and it can exert harmful forces on the supporting teeth and residual ridges. In order to minimize the non physiological forces and preserve the health of the tissues many authors have been focused on biomechanical principles of removable partial dentures design and particularly on the distribution of the excessive forces to the abutment teeth, but also the support, stability, and retention of the denture RPD $[4,8]$.
But there are many other authors who emphasize the importance of the RPD design principle that takes in consideration plaque control especially for caries prevention and periodontal health. These principles make up the hygienic design principles and are characterized by simplicity and openness of the gingival margins [9]. In general textbooks, these two principles are known as the fundamentals of RPD design [2,4,8,9,10]. Another factor to take in consideration is the type of material used in constructing the RPD. In general practice we encounter:

1. Resin-based removable partial dentures

2. Cast-framework removable partial dentures.

In our study we evaluated these principles in different types of partial dentures and grouped by the Kennedy classification of the defects.

\section{Material and Methods}

\subsection{Subjects}

In this study, are taken into observation 53 patients (34 female and 19 male) with a total number of 65 prostheses divided in 28 maxillary and 37 mandibular dentures. The age group of the patients is between 38-70 years of age. 


\subsection{Design of Removable Partial Dentures}

From the 65 partial dentures, 44 were of cast framework base of $\mathrm{Cr}-\mathrm{Co}$, and 21 were from acrylic resinbase. Most of the extension based dentures covered the lingual sides of abutment teeth and their adjacent teeth with plates to minimize denture mobility during function. The methods and materials used depend on the type of the defect.

We have analyzed the type of clasps used and evaluated mobility scale and clinical conditions of the abutment teeth in each case. Four different groups of Kennedy classification, we have used different type of clasps $[1,2,5,6]$.

\subsubsection{Kennedy Class I Defects}

Bilateral posterior edentulous areas. The primary retention is placed on the teeth near the defect. We used clasp type "G" and the indirect retainer is placed in the frontal teeth at their palatial surfaces. The goal is to minimize the deviation of the denture base toward the alveolar ridge mainly while chewing sticky food.

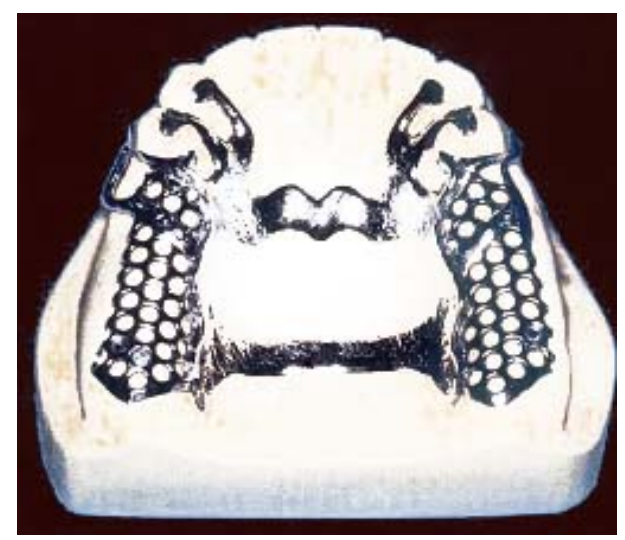

Figure 1. Kennedy Class I defect.

\subsubsection{Kennedy Class II Defects}

Unilateral posterior edentulous area. The primary retention is placed on the teeth near the defect using clasp type "G", and Bonwille clasps at the molars of the other part. In this case as indirect retainer is used a clasp at the first premolar on the opposite side of the defect.

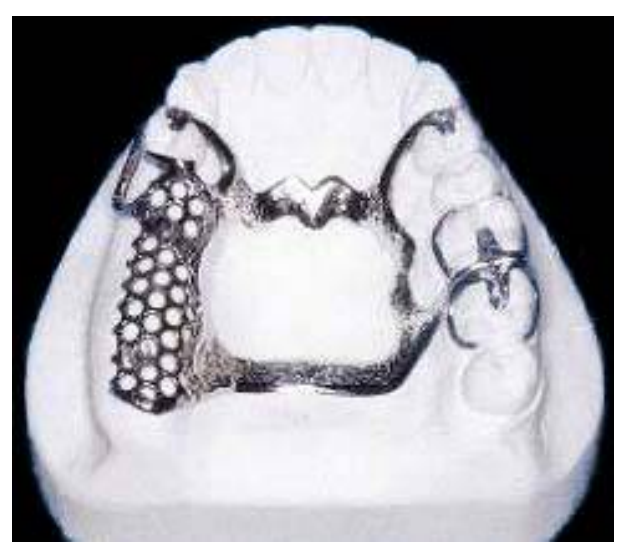

Figure 2. Kennedy Class II defect

\subsubsection{Kennedy Class III Defects}

Unilateral/bilateral posterior edentulous area(s) bounded by remaining teeth. In these cases we have applied clasps type "E". The indirect retainers help stabilize the denture and in this case are used the occlusal clasps placed on the distal teeth.

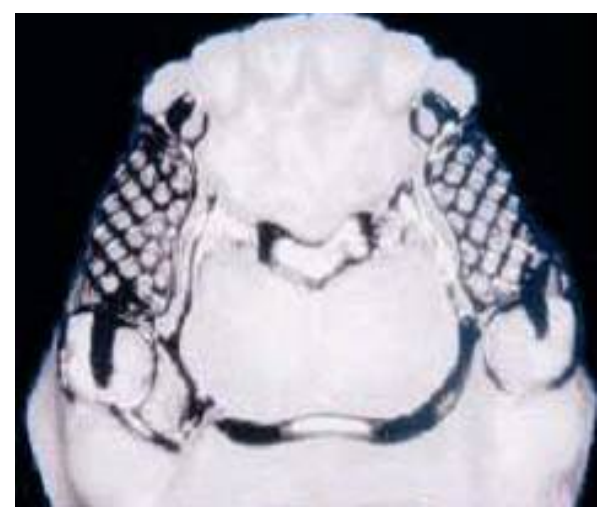

Figure 3. Kennedy Class III defect

\subsubsection{Kennedy Class IV Defects}

Single anterior edentulous area. As primary retentions are used clasps type "E" placed at the first premolars (distally to the defect) meanwhile the indirect retainers are Bonwille clasp applied ate the molars on both sides.

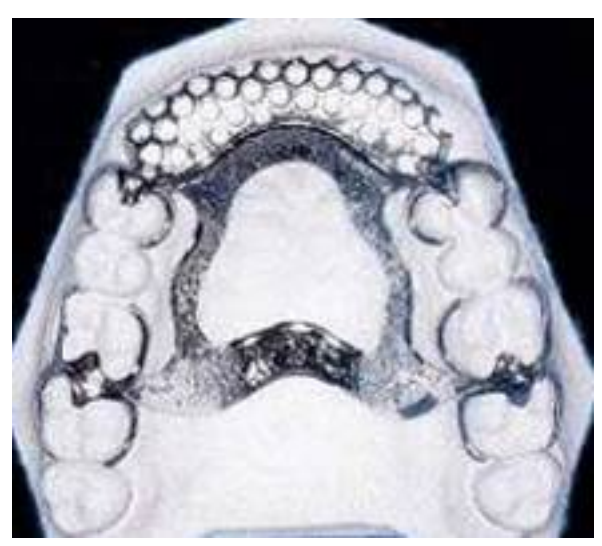

Figure 4. Kennedy Class IV defect

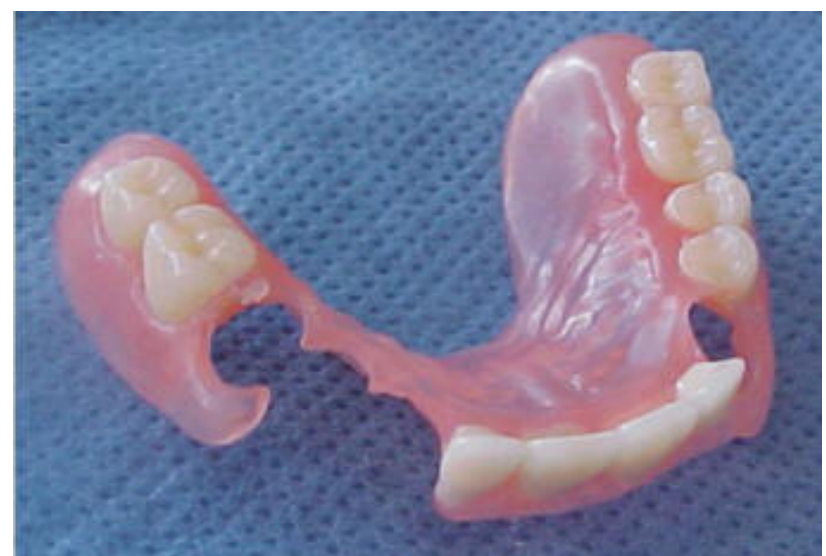

Figure 5. Resin-based RPD

\section{Results}

From the 65 partial dentures applied, only 11 of them resin based dentures and the other 54 were of cast framework Cr-Co. Almost $90 \%$ of the acrylic resin-based dentures required at least 2 extra appointments for 
retouches and after 3 months, 60\% of the patients had discomfort and mobility problems which required our intervention. In one case was recorded fracture of the denture. It permits immediate visualization of the partially edentulous arch and allows easy distinction toothsupported versus tooth-tissue supported prostheses. The patients with cast framework Cr-Co toward those with resin based denture had these differences:

1. Accurate tissue adaptation (better retention).

2. Easy to clean.

3. Strong.

4. Heat conductivity (physiologic tissue stimulation).

On the other hand, the cast framework based dentures showed overall much better stability with no evidence of fractures, of discomfort after the first month. All the model preparations were made using the dental surveyor.

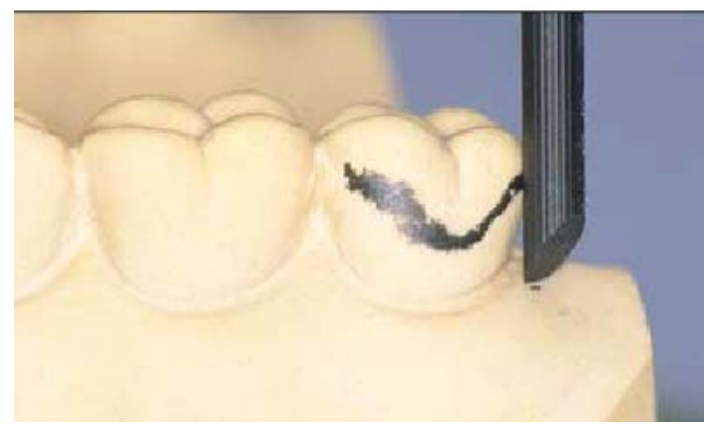

Figure 6. Graphite marker of the surveyor

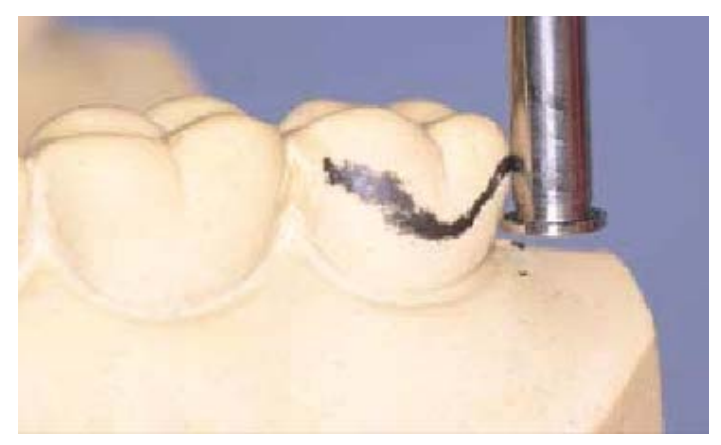

Figure 7. Undercut gauge of the surveyor

The angle formed between the maximal tooth convexity and the cylindrical instrument is called the "angle of gingival convexity". When the convergence angle is less than $10^{\circ}$ the clasp retention cannot be obtained $[3,7,10]$.

The statistical data regarding the cast framework RDPs will be published in a follow-up study after the recall of all the patients and thorough examination of the abutment teeth.

Till now, approximately two years after completing the restorations, no significant evidence of problems regarding our discussion about cast framework RDPs are reported.

\section{Discussion}

Before constructing an RDP, a thorough study is needed. This study must include the evaluation of the type of the defect, evaluation of the different forces that will be applied on the denture and the defining of the retentive elements to be used.
The main forces that the dentures are submitted are the chewing forces. But in the planning of a partial denture it is important to consider also the action of horizontal and rotational forces. The vertical forces are well tolerated by the supporting teeth because the periodontal fibers get into action. It is important that the construction of the denture has a controlled distribution of the force along the axial direction of the tooth (made possible by the supporting arm of the clasp). The forces are also distributed by the retention arm of the clasp and the denture body itself over the alveolar ridge [1,7].

Horizontal forces are harmful forces and applied on the supporting teeth and the alveolar ridge. To minimize their effect is important that the retentive and the stabilizing arm of the clasp to be fitted correctly in the appropriate areas on the tooth and also is important to find the right direction of insertion of the denture itself. Rotational forces may occur in the absence of distal teeth, mainly in Kennedy class I and II $[1,3,8]$. These forces provoke the rotation of the base of the denture over the surface of the edentulous ridge. In this case it is necessary the use of indirect retentions by using supporting arms as far as possible on the other side of the axle of the rotation. Other factors that help minimize their effect are: a wide base of the denture, smaller denture tooth's surface, and by taking a compressed impression during the clinical phase in order to minimize the tissue resilience.

The elimination of the mesial-distal movement is achieved when the denture base has good contact with the proximal surfaces of the supporting teeth and the clasps include the tooth perimeter by over $180^{\circ}$. In the lower jaw these forces are better controlled by including the mandibular tuberculum at the base of the denture.

Cast framework partial dentures have demonstrated a relatively short longevity. In many cases there are reports of $50 \%$ survival rate 10 years after treatment regarding clasp retained removable partial dentures and 50\% survival rate after 4 years for resin based removable partial dentures without cast metal frameworks. These studies do not give particulars regarding the contraindications or faulty design of the RPDs, but they emphasize the necessity to focus on the long-term effects of the restorations. A few studies of cast framework RPDs have shown that with a good design and careful monitoring of the patient, the results can be predictably successful.

\section{Conclusion}

The advantages of cast framework partial dentures made of Cr-Co toward the resin based dentures are:

1. Patient Comfort. Partial dentures constructed with Cr-Co are thin, thus reducing the thickness required for resistance in resin base materials. The reduced thickens provides more sensation and the flexibility of the metal absorbs the shock exerted by the chewing forces. The patient has ease of use from the first day and does not experience difficulties caused by rigid base materials.

2. Durability. These restorations are very durable to compressive and bending forces and do not deteriorate chemically when they come in contact 
with the liquids or bacteria and the chemical environment of the mouth.

3. More biocompatible.

4. Stress-breaking function. The forces exerted on the edentulous ridge and the supporting teeth are substantially reduced.

5. Greater longevity.

6. Increased resistance.

7. Enhanced stability.

8. Better esthetics.

\section{References}

[1] Keraj F - "Protezat parciale” - 2008.
[2] Becker, C. Et al: "Evolution of Removable partial denture design.” J. Prosthodont. 3: 158-66: 1994.

[3] Precisions verankerungen in der zahnarztlichen Prothetik 275-2991983.

[4] Reagan S. Et al - "Practical, aesthetic options for retention of removable partial dentures” 1996; 333-340.

[5] The new Bego - "Partial denture technique" 2002.

[6] Warr J.A. - "Numerical system of clasp design" J. Prosth. Denth. 1961-11.

[7] Watt, MD; McGrgor AR - "Designing partial dentures.” Nosby 1996.

[8] Graken WL - "Partial denture construction" 1995.

[9] Zlataric DK, Čelebić A, Valentić-Peruzović M. "The effect of removable partial dentures on periodontal health of abutment and non-abutment teeth." J Periodontol 2002; 73: 137-144.

[10] Alfonco C. Toothaker; R. W. Wright et. al. "Technique to create appropriate tooth contours for removable partial dentures." 1999; 8: 273-275. 